\title{
Vaccins pour adultes : Le temps est venu
}

\author{
Gemmill I, 2* \\ ${ }^{1}$ Président, Comité consultatif national de l'immunisation \\ ${ }^{2}$ Bureau de santé de Kingston, Frontenac, Lennox et Addington, Kingston (Ontario) \\ *Correspondance : lan.gemmill@kflapublichealth.ca
}

\section{Résumé}

Les bienfaits des vaccins pour adultes ne sont pas appréciés à leur juste valeur, en raison de toute l'attention portée sur les vaccins destinés aux enfants. Toutefois, c'est précisément en raison du succès des programmes d'immunisation des enfants que la plupart des décès attribués à des maladies pouvant être prévenues par un vaccin se produisent aujourd'hui au sein de la population adulte. Les rappels du vaccin antitétanique contribuent à maintenir de faibles taux de tétanos au Canada et les rappels du vaccin contre la coqueluche sont maintenant offerts aux adultes. Le vaccin contre le virus du papillome humain peut être indiqué chez certains adultes plus âgés. Le vaccin contre l'hépatite A et l'hépatite B peut également être indiqué en cas de risque lié à la profession, aux voyages ou au style de vie. Les vaccins contre les infections à pneumocoque ou l'herpès zoster sont recommandés aux personnes âgées de 65 ans et plus et tous les adultes profitent des bienfaits associés à la vaccination annuelle contre l'influenza. Une approche systématique d'immunisation des adultes contribuerait à s'assurer que tous ceux qui sont admissibles à des vaccins particuliers pourraient s'en prévaloir. Cette approche comprendrait la promotion de l'immunisation de routine comme élément fondamental de toute rencontre avec le patient et l'utilisation d'outils, tels qu'un questionnaire sur l'immunisation des adultes et un carnet d'immunisation des adultes de format portefeuille. En investissant dans de telles stratégies, les adultes pourraient jouir d'une bien meilleure santé.

\section{Introduction}

Les vaccins ont énormément amélioré la vie des enfants, en réduisant la morbidité et la mortalité découlant des infections infantiles. Dire qu'avant l'introduction d'un vaccin sûr et efficace, on comptait des dizaines de milliers de cas, lors des épidémies de rougeole, alors qu'aujourd'hui, une poignée de cas dans la plus grande ville canadienne est considérée comme une éclosion importante. La santé des enfants a énormément profité de la mise au point de vaccins sûrs et efficaces.

Cependant, dans cette merveilleuse histoire, les bienfaits des vaccins pour adultes sont sous-estimés. L'immunisation a profité à de nombreuses personnes et amélioré la santé des adultes. Sans être surévaluée, cette importante ressource pour la santé des adultes doit être sans cesse répétée, de très nombreux adultes ne considérant pas la vaccination comme faisant partie des soins à accorder à leur santé ou ne percevant pas toute sa valeur. L'objectif de cet article est de passer en revue les bienfaits des différents vaccins pour adultes et d'examiner de quelle façon ils pourraient faire l'objet d'une promotion encore plus efficace.

\section{Le bien-fondé des vaccins pour adultes}

Plusieurs raisons justifient l'immunisation des adultes. D'abord, une fois leur scolarité terminée, ils semblent oublier la vaccination systématique, mais de nombreux avantages découlent du fait d'assurer l'administration de vaccins à toutes les étapes de la vie. Au Canada, nous avons la chance de pouvoir compter sur un vaccin sûr et efficace qui fait en sorte que les cas de tétanos y sont très rares, alors qu'ailleurs dans le monde, cette maladie affecte encore des milliers de personnes (1). Si cette immunisation n'est plus sur l'écran radar des gens et de leurs fournisseurs de soins de santé, ces bienfaits diminueront avec le temps. Il existe déjà des preuves que, parmi les adultes, la protection sérologique connaît un recul au Canada (2). Le vaccin contre la coqueluche fait partie des immunisations de routine chez les enfants, mais, il y a une dizaine d'années, un vaccin sûr et efficace 
a également été mis au point pour les adultes. II faut toutefois reconnaître qu'amener les gens et les professionnels de la santé à penser au vaccin contre la coqueluche est une autre histoire. Ce vaccin prévient l'apparition de la maladie chez les adultes et son utilisation chez certaines personnes, comme les femmes enceintes, s'est avérée prometteuse, en réduisant la prévalence de la coqueluche chez les plus vulnérables, à savoir les enfants en bas âge (3).

Ensuite, certains vaccins sont recommandés pour les adultes qui présentent des facteurs de risque associés à certaines infections. Par exemple, chaque année, 1500 cas de cancer du col de l'utérus sont diagnostiqués et 400 femmes meurent d'un cancer qu'il est maintenant possible de prévenir. Le vaccin contre le papillomavirus humain (VPH), principale cause du cancer du col de l'utérus, a été approuvé non seulement pour les adolescentes, mais aussi pour les femmes adultes et les hommes de 26 ans et moins. Ce vaccin permet de prévenir $70 \%$ ou plus des cas de cancer du col de l'utérus, plusieurs autres cancers de la région anogénitale et peut même empêcher l'apparition de cancers de la tête et du cou (4). L'homologation et l'administration de ce vaccin nonavalent à grande échelle, associées au dépistage approprié du cancer du col de l'utérus, auraient le potentiel d'éliminer cette importante menace pour la santé des femmes. D'autres vaccins, comme l'immunisation contre l'hépatite $\mathrm{A}(\mathrm{VHA})$ et l'hépatite $\mathrm{B}(\mathrm{VHB})$, réduisent ou éliminent les risques d'infection liés à la profession, aux voyages ou au style de vie.

Certains vaccins sont destinés exclusivement aux adultes. Le vaccin contre le zona fournit une protection individuelle contre la récurrence douloureuse et débilitante de l'herpès zoster. Jusqu'à récemment, le vaccin contre l'influenza n'était pratiquement recommandé qu'aux adultes, afin de prévenir les hospitalisations et les décès dus à la grippe chez les personnes âgées et celles dont la santé est compromise. Son utilisation s'étend désormais plus largement, prévenant une maladie qui peut s'avérer grave chez les femmes enceintes et certains enfants et très désagréable chez les adultes par ailleurs en bonne santé. Depuis plusieurs années, le vaccin polysaccharidique contre le pneumocoque est recommandé à toutes les personnes âgées afin de les protéger des 23 souches de cette affection potentiellement mortelle. Un vaccin conjugué permet aux adultes à haut risque de bénéficier d'une meilleure protection contre 13 souches.

Malgré d'importants bienfaits pour la santé, les vaccins ne font pas partie des priorités des patients et des professionnels de la santé $(5,6)$. D'autres préoccupations revêtent, à juste titre, une grande importance, en matière de santé à l'âge adulte : s'alimenter sainement, avoir une vie active, maintenir un poids idéal et prévenir le diabète et l'hypertension. En raison du succès des programmes d'immunisation des enfants, la plupart des décès attribués à des maladies pouvant être prévenues par un vaccin se produisent aujourd'hui au sein de la population adulte (7). L'immunisation des adultes mérite qu'on lui accorde une plus grande priorité. Elle devrait faire partie des préoccupations des professionnels de la santé lors des rencontres avec leurs patients et, à titre de prestataires de services de santé, nous devrions aider nos patients à mieux comprendre la valeur que peut représenter l'immunisation pour leur santé et les mobiliser comme partenaires dans l'optimisation de ses bienfaits. Parce que les vaccins sont systématiquement offerts à tous les enfants, la prévention de maladies a été couronnée d'un immense succès. Mais l'immunisation des adultes, pour sa part, a souvent été ciblée, entraînant un succès moins éclatant. Par exemple, lorsque le programme de vaccination contre l'influenza est devenu universel en Ontario, en 2000 , certains patients pour qui le vaccin était médicalement indiqué se sont finalement présentés pour être inoculés - pas parce que le vaccin leur était médicalement recommandé, mais parce qu'il était maintenant offert sans frais à tous les résidents de la province.

\section{Promotion des vaccins pour adultes}

Comment peut-on faire de l'immunisation une plus grande priorité dans les programmes de santé des adultes? Première, il faut en reconnaître les bienfaits, tant pour les patients que pour le système de santé. Par exemple, depuis plusieurs années, l'immunisation à l'aide du vaccin polysaccharidique contre le pneumocoque est considérée comme une mesure rentable pour les personnes de plus de 65 ans $(8,9,10)$. Le vaccin contre le zona peut non seulement réduire la souffrance, mais aussi les soins de santé (11). Une approche systématique d'immunisation des adultes contribuerait à s'assurer que tous ceux qui sont admissibles à des vaccins particuliers puissent s'en prévaloir. La première stratégie consiste à changer notre façon de penser au sujet des vaccins pour adultes, de sorte que l'immunisation de routine soit un élément fondamental de chaque rencontre, au même titre que la vaccination des enfants. En effet, ne pas parler d'immunisation lors des visites d'un enfant bien portant serait perçu comme une lacune dans la pratique professionnelle. Pourtant, personne ne critique un médecin qui 
ne s'informe pas de l'état d'immunisation d'un adulte lors d'une visite épisodique, encore moins lors d'un examen de routine.

La promotion de cette portion importante des soins primaires est essentielle si on veut s'assurer que l'immunisation jouit d'une portée universelle et qu'on profite de ses bienfaits.

La deuxième initiative vise à soutenir les prestataires de soins de santé primaires en leur offrant des outils qui leur permettront d'identifier et d'évaluer de façon ciblée les patients qui pourraient être admissibles à une immunisation, afin de s'assurer qu'ils tirent pleinement profit de ces vaccins. Cette stratégie mettrait l'accent, dans un premier temps, sur la détermination de l'admissibilité, en posant les bonnes questions à chaque patient et, deuxièmement, en évaluant l'admissibilité spécifique dans le cadre d'un programme de vaccination ciblée. Cette approche suppose une connaissance des différents vaccins auxquels le patient pourrait être admissible et la capacité de déterminer plus précisément ceux qui sont indiqués. Des outils offrant une information facilement accessible sur le calendrier de routine et à risque élevé (RÉ) pour les adultes (12) et le calendrier de rattrapage pour les adultes sans carnet ou antécédent d'immunisation précisément défini (13) par province et territoire est mise à votre disposition par l'Agence de santé publique du Canada et les ministères de la Santé. Ils doivent être consultés systématiquement.

Des stratégies particulières peuvent aider les prestataires de soins de santé primaires. Par exemple, des procédures s'appliquant à chaque visite des patients pourraient être instaurées dans tous les établissements de soins primaires et comprendre une évaluation de l'état d'immunisation, facilitée au moyen de rappels estampillés dans la fiche des patients ou de rappels automatiques dans les dossiers médicaux électroniques. La tenue des dossiers des patients adultes peut, par ailleurs, être simplifiée, en les intégrant au registre d'immunisation de chaque province et en adoptant des mesures visant à permettre à chaque bureau de soins de santé primaires d'avoir accès à la base de données à distance. Les adultes qui ont fait l'objet d'une évaluation de leur état vaccinal et d'une mise à jour, en recevant les vaccins recommandés, devraient systématiquement obtenir les documents concernant leur immunisation. De nombreux outils, tels que la Fiche de vaccination de l'adulte, proposée par Immunisation Canada, sont déjà prêts et mis à la disposition des prestataires de soins de santé primaires (14). Ces outils comportent également l'avantage d'amener les patients à prendre une part plus active dans leur propre programme de soins et à en savoir plus sur leurs besoins et leur statut en matière de vaccination (15). Le questionnaire sur la vaccination des adultes d'Immunisation Canada, que les patients remplissent à chaque rendez-vous prévu, améliore la documentation sur le patient et la sensibilisation de celui-ci sur son propre état vaccinal.

Autre élément très important pour amener les adultes à s'approprier la responsabilité de leur immunisation : une grande importance accordée à la communication avec les patients. Prendre le temps d'expliquer clairement la valeur de l'immunisation - ainsi que les risques et les bienfaits associés aux différents vaccins - peut contribuer à améliorer la prise vaccinale. Une rémunération appropriée des médecins doit être mise en place pour assurer leur engagement et la réalisation de ce que nous savons tous au sujet du rôle des médecins dans l'amélioration des taux d'immunisation : une communication efficace avec les prestataires de soins de santé primaires influe grandement sur les décisions des gens sur le fait de procéder ou non à leur immunisation (5).

\section{Conclusion}

L'immunisation des adultes est une portion négligée des programmes de vaccination et des soins de santé des adultes. Adoptons une vision partagée d'un programme complet d'immunisation des adultes, un programme au sein duquel : on accorde à la vaccination des adultes l'importance que lui confèrent d'autres initiatives de prévention; on donne la priorité à de nouveaux vaccins efficaces pour les adultes, au même titre que les vaccins pour les enfants; on permet que des vaccins sûrs et efficaces, recommandés aux adultes, soient disponibles dans les provinces et territoires; on met en place une excellente promotion des vaccins pour adultes et, enfin, on s'assure que les efforts se traduisent par une prise vaccinale optimale et une vaste immunisation des adultes, comprenant les rappels nécessaires et les nouveaux vaccins. Avec une approche et des investissements adéquats, la santé des adultes peut être grandement améliorée, grâce à l'attention portée à leur immunisation, exactement comme nous l'avons déjà fait en ce qui concerne la santé des enfants. 


\section{Conflit d'intérêts}

\section{Aucun}

\section{Financement}

Aucun

\section{Références}

(1) World Health Organization (WHO). Vaccine-Preventable Disease Monitoring System, 2014 Global Summary. Global and regional immunization profile.

http://www.who.int/immunization/monitoring_surveillance/data/gs_gloprofile.pdf

(2) Public Health Agency of Canada. Vaccine coverage amongst adult Canadians: Results from the 2012 adult National Immunization Coverage (aNIC) Survey. 2014 Apr 10.

http://www.phac-aspc.gc.ca/im/nics-enva/vcac-cvac-eng.php (disponible en français: http://www.phacaspc.gc.ca/im/nics-enva/vcac-cvac-fra.php).

(3) Healy CM, Baker CJ. Infant pertussis: What to do next? Clinical Infectious Diseases. 2012;54(3):328-30.

(4) National Advisory Committee on Immunization (NACl). Update on Human Papillomavirus (HPV) Vaccines: An Advisory Committee Statement (ACS). CCDR. 2012 Jan:38 ACS-1.

(disponible en en français: http://www.phac-aspc.gc.ca/publicat/ccdr-rmtc/12vol38/acs-dcc-1/index-fra.php).

(5) Johnson DR, Nichol KL, Lipczynski K. Barriers to adult immunization. Am J Med. 2008 Jul;121(7) Suppl 2:S28-35.

(6) Szilagyi PG, Shone LP, Barth R, Kouides RW, Long C, Humiston SG, Jennings J, Bennett NM. Physician practices and attitudes regarding adult immunizations. Preventive Medicine. 2005 Feb;40(2):152-61.

(7) Parkins MD, McNeil SA, Laupland KB. Routine immunization of adults in Canada: Review of the epidemiology of vaccine-preventable diseases and current recommendations for primary prevention. Can J Infect Dis Med Microbiol. 2009 Fall;20(3):e81-90.

(8) Patrick KM, Woolley FR. A cost-benefit analysis of immunization for pneumococcal pneumonia. JAMA. 1981;245(5):473-7.

(9) Mangtani P, Roberts JA, Hall AJ, Cutts FT. An economic analysis of a pneumococcal vaccine programme in people aged over 64 years in a developed country setting. Int J Epidemiol. 2005 Jun;34(3):565-74. Epub 2005 Mar 11.

(10) Castañeda-Orjuela C, Alvis-Guzmán N, Paternina AJ, De la Hoz-Restrepo F. Cost-effectiveness of the introduction of the pneumococcal polysaccharide vaccine in elderly Colombian population. Vaccine. 2011 Oct 13;29(44):7644-50. Epub 2011 Aug 18.

(11) Najafzadeh M, Marra CA, Galanis E, Patrick DM. Cost effectiveness of herpes zoster vaccine in Canada. Pharmacoeconomics. 2009;27(12):991-1004.

(12) Public Health Agency of Canada. Publicly-Funded Immunization Programs in the Provinces and Territories of Canada: Routine and High Risk (HR) Schedule for Adults). 2014 Sep 24.

http://www.phac-aspc.gc.ca/im/ptimprog-progimpt/table-3-eng.php.

(disponible en français: http://www.phac-aspc.gc.ca/im/ptimprog-progimpt/table-3-fra.php).

(13) Public Health Agency of Canada. Publicly-Funded Immunization Programs in the Provinces and Territories of Canada: Catch-Up Schedule for Adults with No Record or Unclear History of Immunization 2014 Sep 24. http://www.phac-aspc.gc.ca/im/ptimprog-progimpt/table-6-eng.php (disponible en français: http://www.phacaspc.gc.ca/im/ptimprog-progimpt/table-6-fra.php).

(14) Immunization Canada. Adult Immunization Record. 2012 May. http://resources.cpha.ca/immunize.ca/data/0104e.pdf

(15) Dubey V, Mathew R, Iglar K, Moineddin R, Glazier R. Improving preventive service delivery at adult complete health check-ups: The Preventive health Evidence-based Recommendation Form (PERFORM) cluster randomized controlled trial. BMC Family Practice. 2006;7:44. 